\title{
High-Resolution Transmission Electron Microscopy on an Absolute Contrast Scale
}

\author{
A. Thust \\ Institute of Solid State Research, Research Centre Jülich, D-52425 Jülich, Germany
}

(Received 21 January 2009; published 4 June 2009)

\begin{abstract}
A fully quantitative approach to high-resolution transmission electron microscopy requires a satisfactory match between image simulations and experiments. While almost perfect agreement between simulations and experiments is routinely achieved on a relative contrast level, a huge mutual discrepancy in the absolute image contrast by a factor of 3 has been frequently reported. It is shown that a major reason for this well-known contrast discrepancy, which is often called Stobbs-factor problem, lies in the neglect of the detector modulation-transfer function in image simulations.
\end{abstract}

DOI: 10.1103/PhysRevLett.102.220801

PACS numbers: 07.78.+s, 42.30.Lr, 68.37.Og

The technique of high-resolution transmission electron microscopy (HRTEM) offers nowadays the possibility to study the atomic configuration of solid state objects with a resolution of around $0.08 \mathrm{~nm}$. Highly accurate information about atomic positions and chemical occupancies at lattice defects and interfaces can be obtained by the quantitative use of this technique [1].

Because of the quantum-mechanical nature of the electron diffraction inside the object and due to the subsequent electron-optical imaging process, any quantitative extraction of object information from HRTEM images requires a justification by accompanying image simulations. Since the introduction of digital image comparison to HRTEM in the early 1990 s, a satisfactory agreement between simulation and experiment could be established only on a relative basis by disregarding the absolute magnitude of the image contrast [2]. The reason for this still common practice was first published by Hÿtch and Stobbs in 1994, who found a remarkably strong discrepancy between the magnitude of the simulated and the experimental image contrast [3]. In their experiment, the dimensionless quantity of the image contrast, which is defined as the standard deviation of the image intensity distribution after normalization of the image mean intensity to unity, was by a factor of 3 too low when compared to image simulations. Since the image motifs were nevertheless widely consistent between simulation and experiment, the problem was regarded mainly as a scaling problem, and became prominent as Stobbs-factor problem, factor of 3 problem, or contrast-mismatch problem. A satisfactory explanation for this long standing problem, which has been meanwhile frequently reproduced and investigated, has not been found so far [4-9].

The electron microscopic imaging process can be subdivided into three stages, of which each can be potentially responsible for the contrast-mismatch problem: (i) the diffraction of the incoming electron wave by the object, (ii) the subsequent electron-optical transfer of the diffracted wave by the electromagnetic lenses, and (iii) the final image acquisition by a camera. Concerning the diffraction part, plasmon and phonon scattering were explicitly ruled out as a reason for the problem [10-12], and moreover, a remarkable discrepancy between measured and simulated diffraction data was not found $[6,8]$. Concerning the optical part, the measurement of coherent aberrations and of partially coherent contrast dampening functions is possible with high accuracy and did not reveal inconsistencies [13-15]. However, little attention has been paid to the final image recording step, which involves the frequency-transfer properties of the camera.

In the work of Hÿtch and Stobbs the modulation-transfer function (MTF) of the photographic film plates used at that time was not considered [3]. It is also remarkable that the most popular image simulation software packages EMS and MACTEMPAS do not allow us to incorporate actually measured MTFs [16,17]. In all, the actual MTFs of the meanwhile used CCD (charge coupled device) cameras were often not considered in the literature related to the Stobbsfactor problem $[7,8]$. Concerning the very rare implementation of actually measured MTFs into image simulations, criticism is allowed: Besides the use of an MTF belonging to a different camera than actually used [5], earlier MTF measurements were often based on the noise method $[9,18]$. However, the noise method is known to yield unrealistic results compared to the knife-edge method, which is the state-of-the-art technique for MTF measurement [19-22].

The drastic influence of the detector MTF on the obtained image contrast is demonstrated here by a simple experiment, where the same area of a $\mathrm{SrTiO}_{3}$ crystal is imaged with different magnification settings of a FEI Titan 80-300 microscope equipped with a $2 k \times 2 k$ Gatan UltraScan 1000 CCD camera of $14 \mu \mathrm{m}$ pixel size. From Table I, and from visual inspection of Fig. 1, the strong dependence of the obtained image contrast on the detector sampling rate is obvious. Although all images are recorded with a sufficiently high sampling rate, a difference in the obtained contrast by a factor of 2 arises between the lowest 
TABLE I. Contrast obtained with a FEI Titan 80-300 microscope equipped with a Gatan UltraScan 1000 camera from an identical area of $\mathrm{SrTiO}_{3}$ using four different microscope magnifications. Nominal magnification, sampling rate, inverse sampling rate, and contrast are listed.

\begin{tabular}{cccc}
\hline \hline $\begin{array}{c}\text { Nominal } \\
\text { magnification }\end{array}$ & $\begin{array}{c}\text { Sampling } \\
\text { rate } \\
{[1 / \AA]}\end{array}$ & $\begin{array}{c}\text { I. Sampling } \\
\text { rate } \\
{[\mathrm{nm}]}\end{array}$ & Contrast \\
\hline 670000 & 6.709 & 0.01491 & 0.082 \\
850000 & 8.532 & 0.01172 & 0.111 \\
1100000 & 11.033 & 0.00906 & 0.136 \\
1400000 & 14.243 & 0.00702 & 0.165 \\
\hline \hline
\end{tabular}

and highest magnified images. Moreover, there is no indication that this effect is already saturated at the highest available magnification of 1400000 .

A quantification of the described phenomenon requires a measurement of the actual camera MTF, which is the Fourier transform of the point-spread function $\operatorname{PSF}(x, y)$. The real-space convolution of the image intensity with the point-spread function $\operatorname{PSF}(x, y)$ is described in Fourier space as a product of the ideal intensity $I(u, v)$ and the modulation-transfer function $\operatorname{MTF}(u, v)$, yielding the detected image intensity $I_{D}(u, v)$, with

$$
I_{D}(u, v)=I(u, v) \operatorname{MTF}(u, v) .
$$

The modulation-transfer function $\operatorname{MTF}(u, v)$ itself can be separated into a rotationally symmetric part $\operatorname{MTF}_{S}(u, v)$ describing the image convolution by the scintillator and its support, and a part describing the convolution over the quadratic area of a single pixel, yielding

$$
\operatorname{MTF}(u, v)=\operatorname{MTF}_{S}(u, v) \operatorname{sinc}(0.5 \pi u) \operatorname{sinc}(0.5 \pi v),
$$

where the Fourier-space coordinates $(u, v)$ are defined in units of the Nyquist frequency.

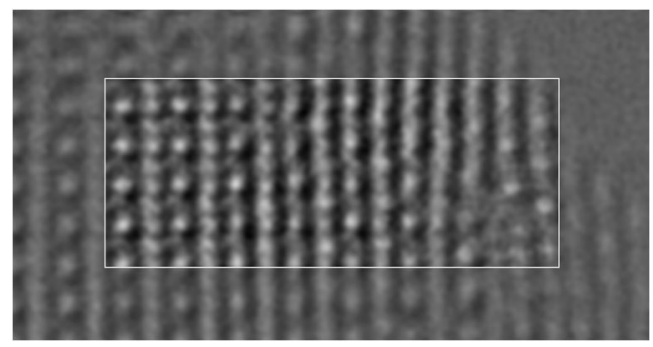

FIG. 1. Edge of a [110]-oriented $\mathrm{SrTiO}_{3}$ crystal recorded with a FEI Titan 80-300 microscope, which is equipped with a Gatan UltraScan 1000 CCD camera. The underlying image was recorded at a nominal electron-optical magnification of 670000 , the inset image at a magnification of 1400000 . For comparison, the images were resampled to identical magnification and are overlaid within the same absolute intensity range between 0.5 (black) and 1.9 (white).
The physical core principle of the MTF measurement used in this Letter is identical to that of the knife-edge method as presented in Refs. [19-21], but is extended from the classical case of a one-dimensional step function to the more general case of a sharply bounded two-dimensional object. Instead of a knife edge positioned directly on the detector, the beam stop located roughly $1 \mathrm{~m}$ above the camera is used as a shadow-forming object. A calculation of Fresnel diffraction for $300 \mathrm{kV}$ electrons falling onto an opaque edge shows that the resulting Fresnel fringing zone at $1 \mathrm{~m}$ distance is much smaller than the pixel size of $14 \mu \mathrm{m}$, thus justifying the assumption of a sharp shadow on the detector. The assumption of full opacity is in turn justified by Monte Carlo simulations, according to which the fraction of registered electrons, which interacted with the edge material, is negligibly small [20].

After correction of the experimental shadow image $I_{E}(x, y)$ for nonuniform illumination, a synthetic shadow image $I_{S}(x, y)$ is constructed in a three-step procedure: First, the experimental image is $n$-fold up-sampled by Fourier interpolation to obtain subpixel positional accuracy. Subsequently, this $n$-fold oversampled image is converted into a sharp two-level image. Finally, this intermediate image is $n$-fold down-binned to obtain a synthetic shadow image $I_{S}(x, y)$ of original size, which is thereby already affected by the pixel-size sinc-convolution of Eq. (2). The experimental and synthetic shadow images differ thus only by the scintillator part $\mathrm{MTF}_{S}$ of Eq. (2). By changing to polar coordinates $(k, \varphi)$ in Fourier-space one can define a set of auxiliary functions, given by

$$
\begin{gathered}
f_{E}(k, \varphi)=\left|I_{E}(k, \varphi)\right|^{2}-N^{2}(k), \\
f_{S}(k, \varphi)=\left|I_{S}(k, \varphi)\right|^{2}, \\
f_{E S}(k, \varphi)=\operatorname{Re}\left\{I_{E}(k, \varphi) / I_{S}(k, \varphi)\right\},
\end{gathered}
$$

where $N^{2}(k)$ is an estimate for the noise power at Fourierspace frequency $k$, and Re denotes the real part. The estimate $N^{2}(k)$ is obtained by evaluating the experimental image $I_{E}(k, \varphi)$ at positions $(k, \varphi)$, where the synthetic image $I_{S}(k, \varphi)$ is known to have no signal contributions. An estimate for $\operatorname{MTF}_{S}(k)$ can then be obtained in two alternative ways, namely, by

$$
\begin{gathered}
\operatorname{MTF}_{S}(k) \approx\left(\left\langle f_{E}\right\rangle /\left\langle f_{S}\right\rangle\right)^{1 / 2}, \\
\operatorname{MTF}_{S}(k) \approx\left\langle f_{E S}\right\rangle,
\end{gathered}
$$

where $\langle f(k, \varphi)\rangle$ denotes a weighted average over the azimuthal coordinate $\varphi$. Within the averaging process, a weighting factor $w(k, \varphi)$ is applied in order to (i) select Fourier-space regions of strong signal known from the synthetic image, (ii) to deselect regions affected by aliasing artifacts following the principle of Ref. [23], and (iii) to deselect regions affected by the periodic continuation artifacts inherent to the Fourier transform. The estimates for 
$\mathrm{MTF}_{S}$ in Eqs. (6) and (7) differ in the sequence of averaging and division, and in the way noise is treated: The estimate of Eq. (6) uses an explicit noise subtraction via Eq. (3), whereas the estimate of Eq. (7) profits from a noise reduction via the real-part projection of Eq. (5). A noticeable difference between the two evaluation modes was not found. A correct noise treatment is crucial, since otherwise noise would be falsely interpreted as signal, yielding a too optimistic MTF.

The result for $\mathrm{MTF}_{S}$, as averaged from ten single measurements, is shown in Fig. 2(c). The most prominent feature of $\mathrm{MTF}_{S}$ is its strong falloff already at low frequencies, which is reflected in the very low central value of the related point-spread function $\mathrm{PSF}_{S}$ shown in Fig. 2(d). The function $\mathrm{PSF}_{S}$ exhibits a wide-ranging tail, which exceeds the area shown in Fig. 2(d), and which is hardly visible there due to its very low level. The ratio $s$ between the central values of the measured PSF and an ideal PSF, which corresponds to the well-known Strehl ratio in light optics [24], adopts a remarkably low value of $s=0.11$. The rapid falloff of the MTF, and the resulting broad PSF, are known to be caused by electrons being backscattered from the scintillator support $[19,25]$.

A focal series of [110]-oriented $\mathrm{SrTiO}_{3}$ was acquired with a spherical-aberration corrected FEI Titan 80-300 microscope operated at $300 \mathrm{kV}$ and is compared with image simulations including the measured MTF. This comparison is intentionally held compact in a sense that a well-known variety of effects, which are known to reduce
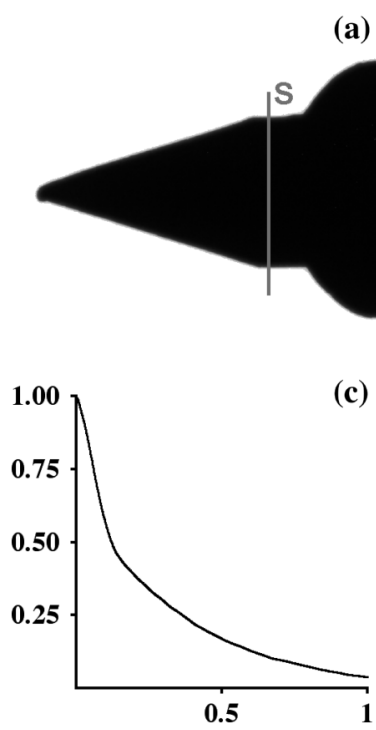

FIG. 2. (a) Shadow image of beam stop acquired with $300 \mathrm{kV}$ electrons by a Gatan UltraScan 1000 camera. (b) Line scan through the vertical section labeled by " $S$ " in the shadow image. Abscissa in pixel units. (c) 1-dim section of modulation-transfer function $\mathrm{MTF}_{S}$ derived from shadow image. Abscissa in units of the Nyquist frequency. (d) Central $9 \times 9$ "pixels" of the related point-spread function $\mathrm{PSF}_{S}$. The bar at the edge shows the height of an ideal $\delta$-like PSF. the image contrast by less than roughly $10 \%$ [4,11,12], like absorption, amorphous cover layers, or phonon scattering, are neglected in the simulations. It is essential to state that a neglect of such effects in simulations has always a tendency to further promote a possibly existing contrastmismatch problem. The series of 30 images was acquired with a spherical aberration of $C_{S}=-25 \mu \mathrm{m}$ at an excessively high sampling rate of 9.71 pixels/Å. Image simulations were carried out with the EMS package [16], using an average Debye-Waller factor of $0.006 \mathrm{~nm}^{2}$ for all atoms within the $\mathrm{SrTiO}_{3}$ unit cell. A beam semiconvergence of $0.25 \mathrm{mrad}$ and a defocus spread of $3.3 \mathrm{~nm}$ were input to the simulations [15]. Special software was devised to inject residual lens aberrations to the EMS wave function $[13,14]$. Residual aberrations, which can occur unintentionally due to a limited lifetime of the perfectly corrected optical state even with a spherical-aberration corrected microscope, were measured directly after recording the series. The contrast dampening effects of the MTF, and of object drift during exposure time, were applied in a final step to the EMS images. Since the effect of object drift is marginal compared to that of the MTF, it will not be separately discussed in the following. The main simulation steps are visualized by four exemplary images in Fig. 3, where an almost perfect match between simulation and experiment is obtained on an absolute contrast scale for an object thickness of $2.8 \mathrm{~nm}$. A quantitative contrast comparison for the complete series is shown in Fig. 4. It can be seen there that the residual aberrations, which change the image motifs in a drastic way, have a comparatively small impact on the image contrast. The major contrast reducing effect is clearly due to the MTF.

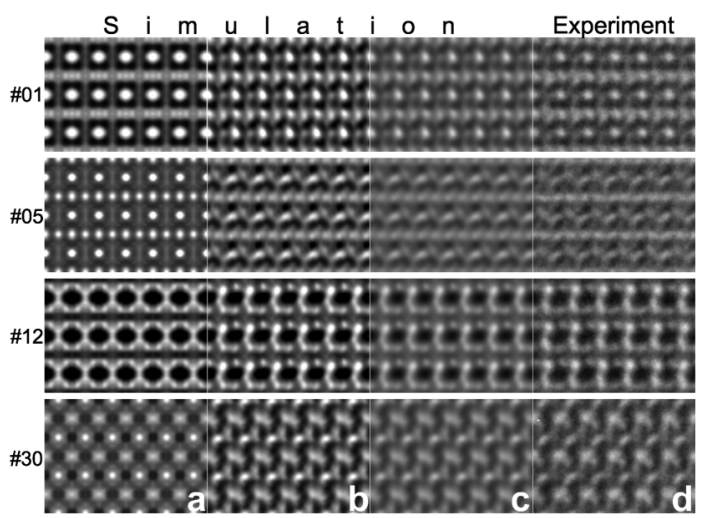

FIG. 3. Image patches comprising $3 \times 3 \quad \mathrm{SrTiO}_{3}$ unit cells projected along the [110] zone axis. The patches 1, 5, 12, and 30 , belong to different focal values and are shown exemplarily for a series of 30 images. All patches are displayed on the same gray scale extending between intensity values 0.35 (black) and 2.2 (white). (a) Simulation for an object thickness of $2.8 \mathrm{~nm}$, (b) simulation including additionally residual lens aberrations, (c) simulation including residual aberrations and additionally the measured MTF, (d) experimental images. 


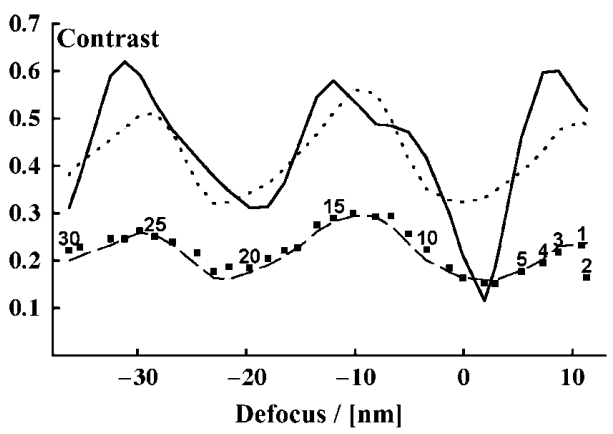

FIG. 4. Solid line: Image contrast simulated for an object thickness of $2.8 \mathrm{~nm}$ and for the focal values of the experimental $\mathrm{SrTiO}_{3}$ series. Dotted line: Simulated contrast including additionally residual lens aberrations. Dashed line: Simulated contrast including residual aberrations and additionally the MTF. Squares: Experimental image contrast. Image numbers within focal series are indicated.

It is important to confirm that the object thickness of $2.8 \mathrm{~nm}$, which was used for the simulations, is indeed unique. Otherwise one could argue that simulated highcontrast images could exist, which result from larger object thickness values, and which could again cause a Stobbsfactor problem. Fortunately, the image motifs resulting from higher simulated object thickness values do not reproduce the experimentally recorded motifs. Since the thickness change of the image motifs cannot be shown here due to space restrictions, a more compact way is chosen to confirm the correct thickness value. Curves displaying the simulated image contrast in dependence of defocus have been calculated for different object thicknesses, including the effect of residual aberrations, and of the MTF. Such exemplary contrast-defocus curves (CD curves) are displayed in Fig. 5(a). These CD curves change their shape in a very characteristic way with object thickness and serve thus as a unique fingerprint of the respective thickness. The shape change of the CD curves is caused by the different scattering behavior of the in-

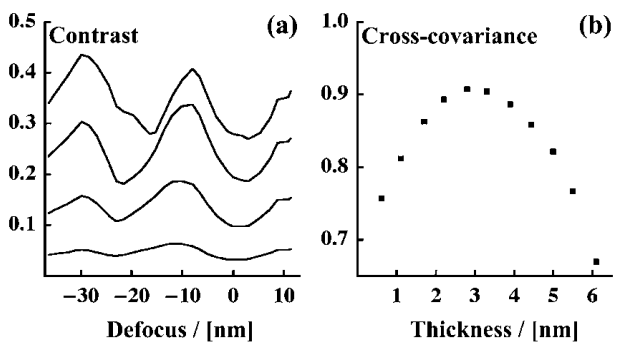

FIG. 5. (a) Simulated image contrast of $\mathrm{SrTiO}_{3}$ including residual aberrations and MTF versus defocus (CD curves). The curves belong to object thickness values of $0.6,1.7,3.3$, and $6.1 \mathrm{~nm}$ (bottom to top). (b) Cross covariance between experimental CD curve (squares in Fig. 4) and CD curves simulated for the object thickness values indicated on the abscissa. volved $\mathrm{Sr}-\mathrm{O}$, Ti, and $\mathrm{O}$ atom columns with object thickness. A comparison between the experimentally recorded $\mathrm{CD}$ curve and the simulated $\mathrm{CD}$ curves based on the mutual cross covariance, which neglects explicitly the absolute contrast scale, and which is purely focused on the curve shape, reveals a maximum curve resemblance at an object thickness of $2.8 \mathrm{~nm}$ [Fig. 5(b)]. Absolute and relative contrast measures are thus fully consistent.

In summary, the existence of a general contrastmismatch problem of remarkable size in HRTEM cannot be confirmed. On the contrary, an almost perfect consistency between simulation and experiment is found when including the correct detector MTF in image simulations.

The author is grateful to Dr. J. Barthel for leaving him the focal series and for recording the shadow images.

[1] K. W. Urban, Science 321, 506 (2008).

[2] A. Thust and K. Urban, Ultramicroscopy 45, 23 (1992).

[3] M. J. Hÿtch and W. M. Stobbs, Ultramicroscopy 53, 191 (1994).

[4] C. B. Boothroyd, J. Microsc. 190, 99 (1998).

[5] C. B. Boothroyd, Ultramicroscopy 83, 159 (2000).

[6] R. Rosenfeld, Ph.D. thesis, Berichte des Forschungszentrums Jülich, 2001.

[7] A. Howie, Ultramicroscopy 98, 73 (2004).

[8] W. Qu, C. Boothroyd, and A. Huan, Appl. Surf. Sci. 252, 3984 (2006).

[9] K. Du, K.v. Hochmeister, and F. Phillipp, Ultramicroscopy 107, 281 (2007).

[10] C. B. Boothroyd, Scanning Microsc. 11, 31 (1997).

[11] C. B. Boothroyd and M. Yeadon, Ultramicroscopy 96, 361 (2003).

[12] C. B. Boothroyd and R.E. Dunin-Borkowski, Ultramicroscopy 98, 115 (2004).

[13] A. Thust et al., Ultramicroscopy 64, 249 (1996).

[14] S. Uhlemann and M. Haider, Ultramicroscopy 72, 109 (1998).

[15] J. Barthel and A. Thust, Phys. Rev. Lett. 101, 200801 (2008).

[16] P. A. Stadelmann, Ultramicroscopy 21, 131 (1987).

[17] M. A. O'Keefe and R. Kilaas, Scanning Microsc. Suppl. 2, 225 (1988)

[18] J. M. Zuo, Microsc. Res. Tech. 49, 245 (2000).

[19] R. R. Meyer and A. Kirkland, Ultramicroscopy 75, 23 (1998).

[20] R. R. Meyer and A.I. Kirkland, Microsc. Res. Tech. 49, 269 (2000).

[21] R. R. Meyer et al., Ultramicroscopy 85, 9 (2000).

[22] R. Henderson et al., in Proceedings of the 14th European Microscopy Congress, Aachen, Germany, 2008 (SpringerVerlag, Berlin, Heidelberg, 2008), Vol. 1, p. 71.

[23] W. J. de Ruijter, Micron 26, 247 (1995).

[24] K. Strehl, Z. Instrumentenkd. 22, 213 (1902).

[25] G. McMullan et al., in Proceedings of the 14th European Microscopy Congress, Aachen, Germany, 2008 (SpringerVerlag, Berlin, Heidelberg, 2008), Vol. 1, p. 73. 